\title{
Autoregulation of convergent RNAi genes in fission yeast
}

\author{
Monika Gullerova, ${ }^{1}$ Danesh Moazed, ${ }^{2}$ and Nick J. Proudfoot ${ }^{1,3}$ \\ ${ }^{1}$ Sir William Dunn School of Pathology, University of Oxford, Oxford OX1 3RE, United Kingdom; ${ }^{2}$ Department of Cell Biology, \\ Harvard Medical School, Boston, Massachusetts 02115, USA
}

RNAi plays a central role in the regulation of eukaryotic genes. In Schizosaccharomyces pombe fission yeast, RNAi involves the formation of siRNA from dsRNA that acts to establish and maintain heterochromatin over centromeres, telomeres, and mating loci. We showed previously that transient heterochromatin also forms over $S$. pombe convergent genes (CGs). Remarkably, most RNAi genes are themselves convergent. We demonstrate here that transient heterochromatin formed by the RNAi pathway over RNAi CGs leads to their autoregulation in G1-S. Furthermore, the switching of RNAi gene orientation from convergent to tandem causes loss of their G1-S down-regulation. Surprisingly, yeast mutants with tandemized dcr1, ago1, or clr4 genes display aberrant centromeric heterochromatin, which results in abnormal cell morphology. Our results emphasize the significance of gene orientation for correct RNAi gene expression, and suggest a role for cell cycle-dependent formation of RNAi CG heterochromatin in cellular integrity.

[Keywords: RNAi genes; autoregulation; convergent genes; fission yeast; heterochromatin]

Supplemental material is available for this article.

Received December 7, 2010; revised version accepted January 25, 2011.

Eukaryotic transcription is known to generate far more transcripts than those simply ascribed to protein-coding or structural RNA genes. Modern genomic analysis reveals that both intergenic and antisense genic transcripts are widespread (Wilhelm et al. 2008; Mattick 2009; Neil et al. 2009; $\mathrm{Xu}$ et al. 2009). While the function of these noncoding (nc) transcripts remains largely unknown, one of their critical roles may be in determining whether chromatin is active or repressed through the operation of RNAi mechanisms (Carthew and Sontheimer 2009). In fission yeast centromeres, this involves the formation of siRNA from dsRNA by Dicer (Grewal and Jia 2007), which then activates the Argonaute-containing transcription silencing complex (RITS). siRNA-activated RITS then associates with target chromosomal regions possessing nucleosomes kept underacetylated by histone deacetylases (HDAC). Consequently, CLCR complex initiates histone H3K9 methylation to form stable heterochromatin (Verdel et al. 2004). The RNA-dependent RNA polymerase complex RDRC also associates physically with this chromatin and is required to maintain heterochromatin (Volpe et al. 2002; Motamedi et al. 2004; Sugiyama et al. 2005). Heterochromatin thus assembled can spread along the chromatin fiber by a process involving proteins

${ }^{3}$ Corresponding author.

E-MAIL nicholas.proudfoot@path.ox.ac.uk; FAX 44-1865-275556.

Article published online ahead of print. Article and publication date are online at http://www.genesdev.org/cgi/doi/10.1101/gad.618611. Freely available online through the Genes \& Development Open Access option. such as Swi6/HP1 (Noma et al. 2001). This spreading may be blocked by specific boundary elements (Sun and Elgin 1999; Noma et al. 2001; AG West et al. 2004) and, in Schizosaccharomyces pombe, by the jmjC domain-containing protein Epe1 (Zofall and Grewal 2006). Critical RNAi components such as Ago1, Dcr1, and Rdp1 are known to be required for centromere function through heterochromatin formation. Thus, their deletion disrupts chromosome segregation leading to a high incidence of lagging chromosomes in late anaphase (Volpe et al. 2003).

While many ncRNAs derive from cryptic promoter activity, some also derive from readthrough transcription caused by the failure of protein-coding genes to terminate transcription. RNA polymerase II (Pol II) termination in eukaryotes is a surprisingly complex process (Proudfoot 2004; Richard and Manley 2009). When elongating Pol II encounters functional polyA signals, these trigger cotranscriptional cleavage and polyadenylation of the premRNA, resulting in the release of mRNA from its site of synthesis on chromatin. Concomitantly, the $3^{\prime}$ product of this RNA processing reaction is rapidly degraded by nuclear 5'-3' exonuclease, which effectively follows the still elongating Pol II complex (S West et al. 2004; West et al. 2008). Transcription termination is then elicited when the nascent RNA is degraded up to the still elongating polymerase and somehow evicts it from the DNA template. Termination may be facilitated by transcriptional pausing, which effectively acts to provide more time for RNA exonucleolytic degradation to occur (Gromak et al. 2006). While this process is relatively well documented in 
Saccharomyces cerevisiae and mammalian cells, little information about the mechanism of 3 '-end processing and termination is available for fission yeast. Of the few genes so far examined-which include ura4, nmt1, and $n m t 2-i t$ is apparent that both specific polyA signals and transcriptional pause sites may be present (Birse et al. 1997; Hansen et al. 1998; Aranda and Proudfoot 1999). In mammalian genomes, gene transcription will often generate multiple mRNA species that use both gene-proximal as well as additional more distal polyA signals (Tian et al. 2005; Sandberg et al. 2008; Mayr and Bartel 2009). This readthrough transcription may therefore have biological importance through the generation of multiple mRNAs from one gene that may have particular regulatory functions. Interestingly, readthrough transcription has also been shown to occur for some genes in S. pombe, in particular when they are arranged in convergent orientation along the chromosome (Gullerova and Proudfoot 2008).

Studies in fission yeast have established that centromeric heterochromatin, thought to be normally transcriptionally silent, paradoxically depends on its own transcription. During mitosis, H3K9 methylation and Swi6 are lost, which allows centromeric transcripts to briefly accumulate in the $S$ phase. Rapid processing of these transcripts into siRNA restores H3K9 methylation and Swi6 occupancy, leading to cohesin recruitment in G2 (Chen et al. 2008; Kloc and Martienssen 2008; Kloc et al. 2008). We also showed that heterochromatin is formed transiently over cotranscribed convergent genes (CGs) in G1-S, as during this phase of the cell cycle readthrough transcription appears to predominate. Overlapping complementary transcripts so generated result in dsRNA formation, which activates the RNAi pathway. CG heterochromatin is recognized by Swi6, which in turn leads to cohesin recruitment (Gullerova and Proudfoot 2008). Cohesin thus localized between CGs throughout the genome (Lengronne et al. 2004) acts to block further transcriptional readthrough in G2. H3K9me3 is subsequently removed during the $\mathrm{S}$ phase, so that CG transcription in G2 now generates nonoverlapping mRNA using proximal polyA sites (Gullerova and Proudfoot 2008). The fission yeast genome contains 5027 proteinencoding genes (Sanger Institute), of which $\sim 30 \%$ are convergent (Singleton and Levin 2002). However, only a fraction of CG pairs may be cotranscribed to produce dsRNA in G1-S.

In this study, we extend our previous analysis of CG expression in fission yeast (Gullerova and Proudfoot 2008) by characterizing the cell cycle expression and heterochromatin formation of CGs that encode (as one partner) RNAi factors. Significantly, all protein complexes currently known to be involved in the RNAi pathway have CG components where the gene pairs in question are cotranscribed. We first show that CGs, unlike tandem genes (TGs), are generally down-regulated in G1-S, and this down-regulation requires the RNAi pathway. We then demonstrate that conversion of three key RNAi genes from convergent to tandem orientation causes loss of their G1-S down-regulation. Surprisingly, this in turn restricts centromeric transcription and causes aberrant cell morphology. Our studies therefore provide evidence for the critical importance of RNAi gene orientation in the correct regulation of heterochromatin formation and, consequently, in cell cycle progression.

\section{Results}

In our previous studies on CGs in S. pombe (Gullerova and Proudfoot 2008), we defined a cell cycle pathway for CG expression that is based on the regulated termination of transcription between CG pairs. In G1, readthrough transcription occurs, while in G2, efficient termination dominates using the gene proximal pA signal. This switch in termination profile is brought about by formation of G1-restricted CG dsRNA, which in turn elicits transient heterochromatin formation, including cohesin deposition. Cohesin then acts as a termination factor in G2, preventing further readthrough transcription and dsRNA formation. Effectively, CG heterochromatin is G1-restricted. This intricate regulatory pathway of CG transcription may in part relate to the need for cohesin recruitment along chromosome arms. Possibly, the restricted recruitment of cohesin solely to centromeres and telomeres is too limited to fully facilitate sister chromatid alignment in G2. However, we wondered whether some CGs might also use this complex transcription regulation for other biological purposes. We therefore scrutinized the genes that exist in convergent orientation to look for potential functional patterns. This led us to the realization that many genes encoding RNAi factors are themselves convergent.

\section{Convergent RNAi genes are down-regulated in G1-S}

Most RNAi genes, which are required for heterochromatin establishment, are themselves convergent (Fig. 1A). RNAi components (Dcr1, Stc1, ARC, RITS, RDRC, CLRC, and HDACs) derive from predominantly convergent genes. On a random basis, it is predicted that CGs represent $\sim 30 \%$ of all RNAi genes. However, they are actually $80 \%$ of all known RNAi genes required for heterochromatin establishment (Supplemental Fig. 1). We analyzed three other randomly selected genetic pathways: polyadenylated mRNA export from nucleus, maintenance of DNA repeat elements, and response to caffeine. In all three cases, CGs represent the expected $25 \%-36 \%$ level of genes involved in these different pathways (Supplemental Fig. 1), arguing against bias in the much higher level of RNAi CGs. The fact that all of these RNAi genes and their CG partners are also cotranscribed (data not shown) further emphasizes the nonrandom CG arrangement of RNAi genes.

Since cotranscribed CGs form transient G1-S heterochromatin, we wished to determine whether such heterochromatin marks result in down-regulation of CG RNAi gene expression. Indeed, global analysis of $S$. pombe gene transcript levels throughout the cell cycle (Sanger Center/ gene expression viewer) shows a mild but consistent reduction in G1-S transcript levels for convergent RNAi 
A

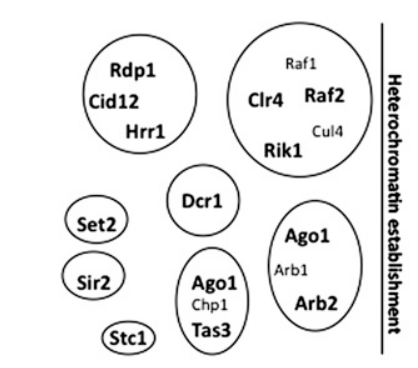

C

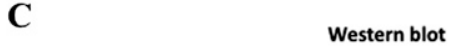

B

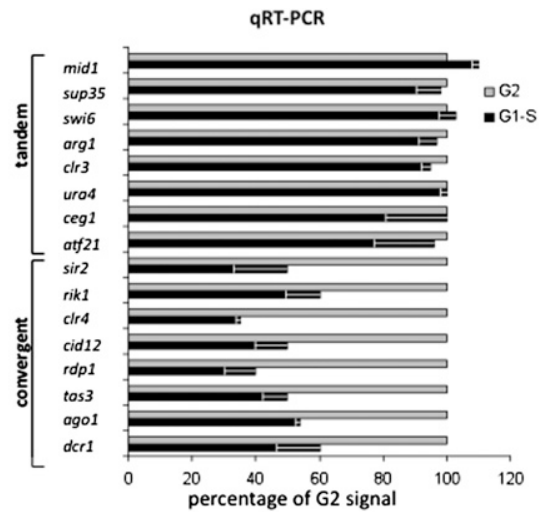

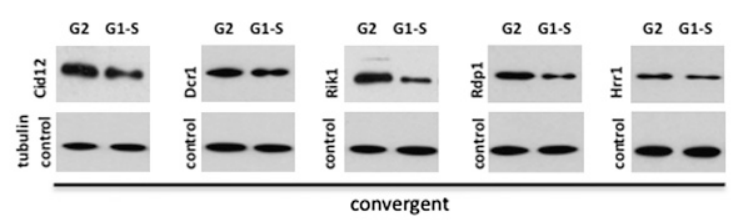
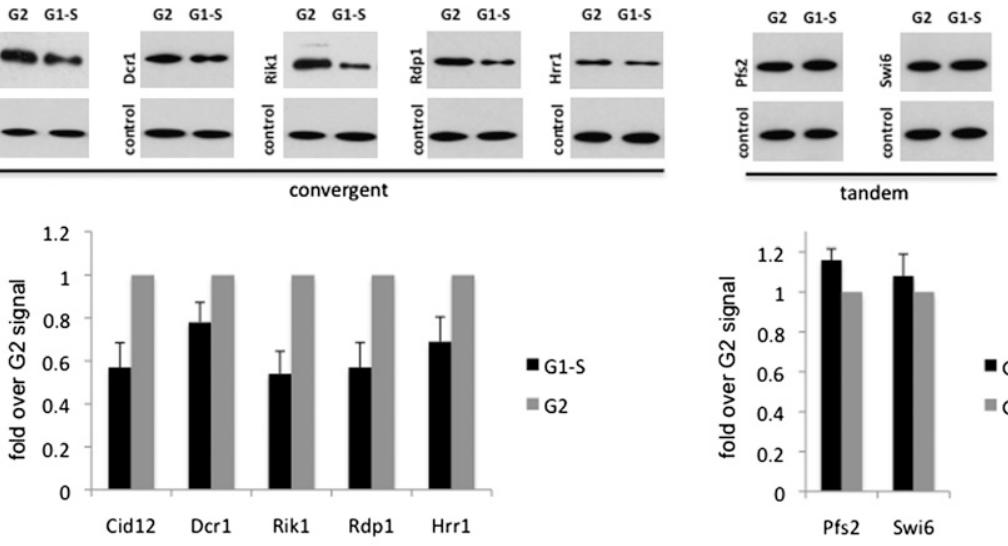

Figure 1. RNAi genes are convergent and down-regulated during G1-S. (A) All RNAi complexes derive from at least two CGs. RNAi CGs are in bold. (B) qRT-PCR analysis shows transcript levels of RNAi CGs in G1-S. Cells were blocked in G1-S by HU (Supplemental Fig. 3A) and compared with cycling (predominantly G2) cells. Eight randomly selected TGs were used as controls. (C) Western blot analysis of RNAi proteins in G1-S and G2. Total protein extracts were isolated from strains expressing RNAi genes with endogenous epitope tags (TAP or Flag). Equal amounts of proteins were loaded on the membrane and detected with either horseradish peroxidase-conjugated anti-peroxidase specific for TAP or horseradish peroxidase-conjugated anti-Flag M2. pfs2-GFP and swi6-GFP TGs were used as controls, detecting their protein expression using anti-GFP. These showed no significant difference between G1-S and G2. Equal amounts of proteins loaded were confirmed by Western blotting using antitubulin antibody. Quantitation was based on three independent experiments. $(D)$ Pol II preferentially localizes to centromeres (cen) in G1-S and to CGs in G2. ChIP signals were normalized to $100 \%$. Relative ratios between G1-S and G2 signals are shown graphically. Centromeric PCR primers detect the dg repeat sequence.

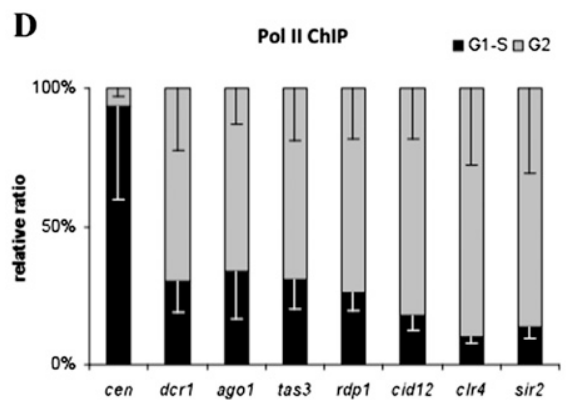

genes. We repeated this analysis using quantitative RTPCR (qRT-PCR) in wild-type G1-S and G2 cells. All CG RNAi mRNA levels decreased in G1-S about twofold as compared with control TGs (Fig. 1B). Similarly, RNAi protein levels were reduced in G1-S blocked cells. The levels of reduction varied from 1.5-fold to twofold, presumably depending on relative protein stability. Note that two TG-derived proteins do not show G1-S-phase reduction in levels (Fig. 1C). We next measured Pol II occupancy over the centromere (cen), CGs, and TGs. As expected, cen Pol II signals were detected in G1-S-phase cells but not in G2 (Fig. 1D). This result correlates with the previously described cell cycle control of centromeric transcription (Chen et al. 2008). However, all tested RNAi CGs showed an opposite effect to cen with higher Pol II levels in G2, but lower Pol II levels in G1-S (Fig. 1D). Notably, the reduction in Pol II levels for CGs in G1-S as compared with G2 was significantly larger than the RNA level change, indicating that nascent transcription is severely affected (by more than fourfold). Finally, we showed that Pol II occupancy over a panel of TGs did not alter significantly through the cell cycle (Supplemental Fig. 2A).

\section{Transient CG heterochromatin requires Dcr1 and Ago1 but not Rdp1 activities}

Since CG and centromeric heterochromatin show different cell cycle regulation, we wished to determine whether this might reflect different usage of the cellular RNAi apparatus. We first verified that heterochromatin forms on CGs using G1 chromatin, as described previously (Gullerova and Proudfoot 2008). Thus, hydroxyurea (HU)-treated wild-type or cdc10ts, mutant cells both blocked in late G1-S, gave similar levels of H3K9me3 
signal over a range of CGs, while no signal was detectable over a TG panel (Supplemental Fig. 3). Previously, temperature shift was shown to cause accumulation of siRNA through inhibition of RNAi (Kloc et al. 2008). Therefore, we elected to use HU-blocked and released cells for further experiments. Using HU-blocked $\Delta d c r 1$, $\Delta a g o 1$, and $\Delta c h p 1$ cells, we observed a near complete loss of heterochromatin marks for CGs (Fig. 2A), confirming a general requirement for Dcrl and RITS complex in heterochromatin formation. Similarly, G2 cen heterochromatin requires Dcrl and RITS. In contrast, gene deletion of all RDRC components had little effect on CG H3K9me3 levels, but a substantial effect on cen (Fig. 2B). Next, we examined the methyltransferase complex CLRC. The H3K9me3 mark was lost at both cen and CGs with $\Delta$ clr4 and $\Delta$ rik1 mutants (Fig. 2C), confirming the crucial role of CLRC in H3K9 methylation. Since nucle- osomes must be kept underacetylated at sites of heterochromatin formation, we also examined the role of HDACs Clr3 and Sir2 in CG heterochromatin formation. While $\Delta$ clr3 gave little reduction of $\mathrm{H} 3 \mathrm{~K} 9 \mathrm{me} 3$ on both cen and $\mathrm{CG}, \Delta$ sir2 led to a substantial decrease in H3K9me3 over cen and its total loss over CGs (Fig. 1D). This confirms that Sir2 is the major H3K9-specific HDAC (Shankaranarayana et al. 2003), essential for heterochromatin formation over CGs.

As shown above, Dcrl and RITS complex are required for CG heterochromatin formation. This implies that CG readthrough transcription forms dsRNA, which is then processed into siRNAs. However, genomic RNA sequencing of siRNAs in $S$. pombe failed to detect significant CG siRNA above background levels of genomewide RNA degradation products (Buhler et al. 2008). Possibly, the transient (G1-S-restricted) nature of CG
$\mathbf{A}$

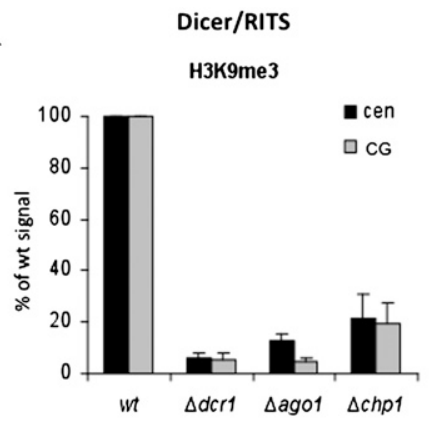

C

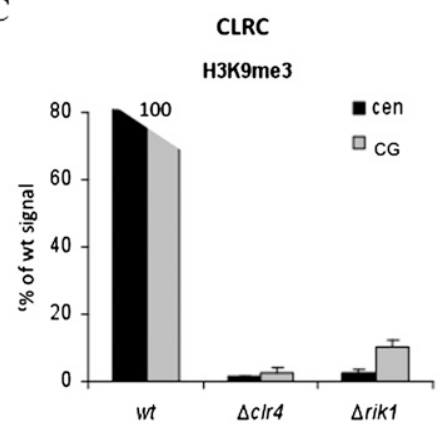

$\mathbf{E}$

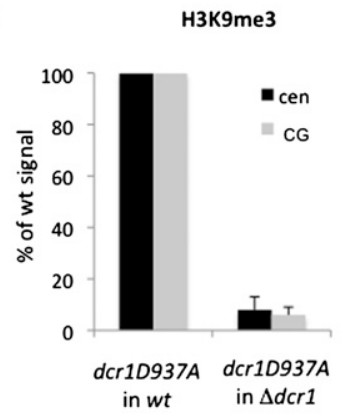

Dcr1

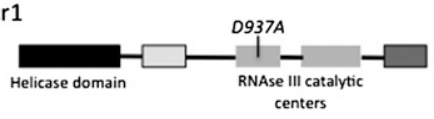

B

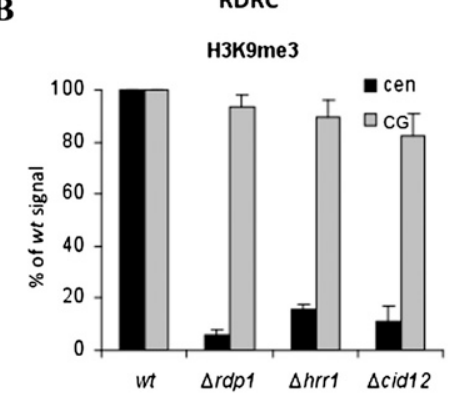

D

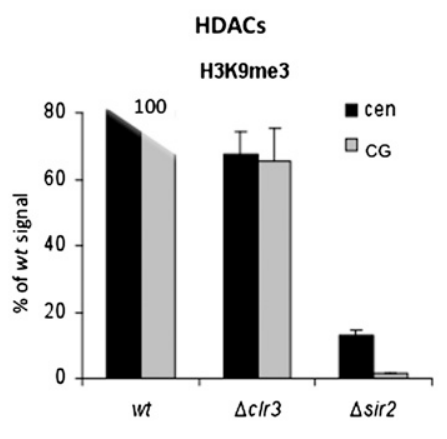

F

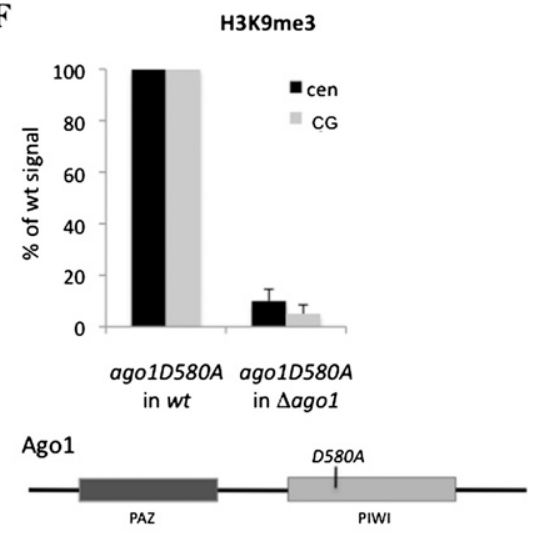

Figure 2. Comparison of heterochromatin (H3K9me3) between cen and CGs. CG heterochromatin was isolated from HUblocked cells (Supplemental Fig. 3A), while cen was from unsynchronized cells. ChIP analysis was performed using $\mathrm{H} 3 \mathrm{~K} 9 \mathrm{me} 3$ antibody. Probe for cen is specific to $\mathrm{dg}$ repeat. Probes for CGs represent average signal of four different CG probes in ORFs (mei4, act1, nmt2, and avn2). ChIP signals were normalized to wild-type levels $(100 \%)$. Bars represent at least three biological repeats. Errors were determined by SD. $(A-D)$ H3K9me3 ChIP analysis is shown on cen and CGs in $\Delta d c r 1, \Delta a g o 1$, and $\Delta \operatorname{chp} 1(A)$, in RDRC deletion mutants $(B)$, in CLRC deletion mutants $(C)$, and for sir2 and clr3 HDAC deletion mutants $(D)$. (E) Plasmid expressing dcr1 D937A (RNase III catalytic domain mutant) was transformed into a $\Delta d c r 1$ background and compared with wildtype cells by ChIP analysis, as above. $(F)$ Plasmid expressing ago1 D580A (slicer mutant) was transformed into $\triangle a g o 1$ and compared with wild-type cells by ChIP analysis, as above. 
heterchromatin, and the fact that no Rdp1 amplification of CG transcription occurs, makes detection of CG siRNA problematic. Even so, CG siRNAs have been detected for some Drosophila CGs (Czech et al. 2008). In view of the very low levels of CG siRNA in $S$. pombe, we tested whether CG heterochromatin depends on Dcrl and Agol RNA processing activity. To do this we used a $d c r 1 D 937 A$ strain mutated in the catalytic site of the RNase IIIa domain (Colmenares et al. 2007), and ago1D580A mutated in the endonucleolytic cleavage or slicing domain PIWI (Irvine et al. 2006; Buker et al. 2007). Significantly, both of these mutants showed loss of centromeric and CG heterochromatin (Fig. 2E,F). We conclude that both dcr1 and ago1 RNA processing activities are required for CG heterochromatin formation. Recent experiments described below (Fig. 5C, below) now show the detection of low levels of G1-S-specific siRNAs from the ago1-mmi1 CGs. This result confirms the requirement of Dcrl and Ago1 RNA processing for CG heterochromatin formation.

\section{RNAi genes are autoregulated in G1-S}

Our above results show that many RNAi genes are convergent and are down-regulated in G1-S by forming transient heterochromatin through the RNAi pathway. We therefore confirmed that RNAi genes are effectively autoregulated by the RNAi pathway during the cell cycle. First, we measured mRNA levels of RNAi CGs from G1-S blocked $\Delta d c r 1, \Delta a g o 1, \Delta c l r 4$, and $\Delta$ sir2 cells and observed variable mRNA accumulation (Fig. 3A), in marked contrast to eight TGs (Supplemental Fig. 2B). According to our above results (Fig. 2B), RDRC is not involved in heterochromatin formation over CGs. Significantly, mRNA levels of RNAi genes in G1-S phase were unaffected in RDRC mutants $\Delta r d p 1, \Delta h r r 1$, and $\Delta c i d 12$ (Fig. 3B). We also quantified Agol levels on chromatin from G1-S blocked and G2 cells. Centromeres showed higher Agol occupancy in G2, but lower levels in G1-S. Strikingly, all tested convergent RNAi genes showed an opposite profile, with high Agol levels in G1-S and only background levels in G2 (Fig. 3C). Notably, we detected only background Agol levels on TGs in both G1-S and G2 (Supplemental Fig. 2C). These results suggest that convergent RNAi genes result in readthrough mRNAs in G1-S that are then processed by RNAi to form their observed transient heterochromatin marks. In an almost mirror-image arrangement, centromeric heterochromatin is fully established throughout G2 with complete transcriptional silencing, while in G1-S, centromeric Pol II and associated transcripts become clearly detectible. The opposite cell cycle regulation of these two heterochromatic classes suggests an autoregulatory process whereby CG RNAi gene down-regulation in G1-S affords some relaxation in centromeric heterochromatin with consequent transcription during this limited cell cycle time period.

\section{Switching convergent RNAi genes}

to tandem orientation

The marked difference in expression patterns of CGs and TGs during the cell cycle suggests that the convergent
A

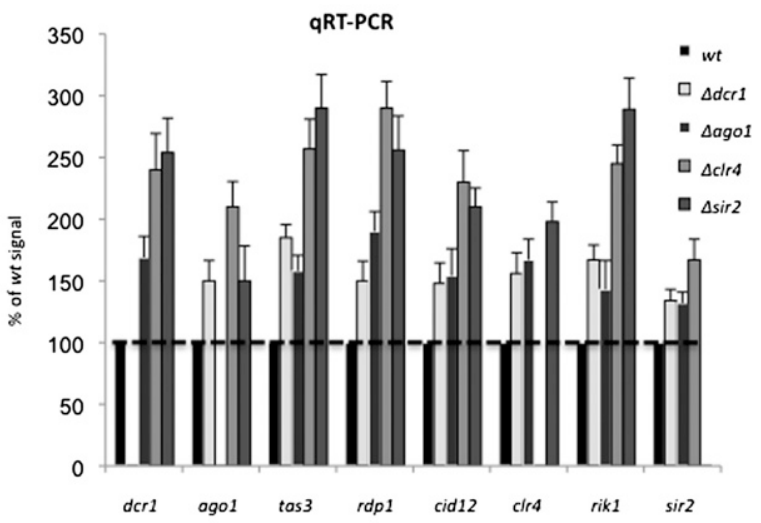

B

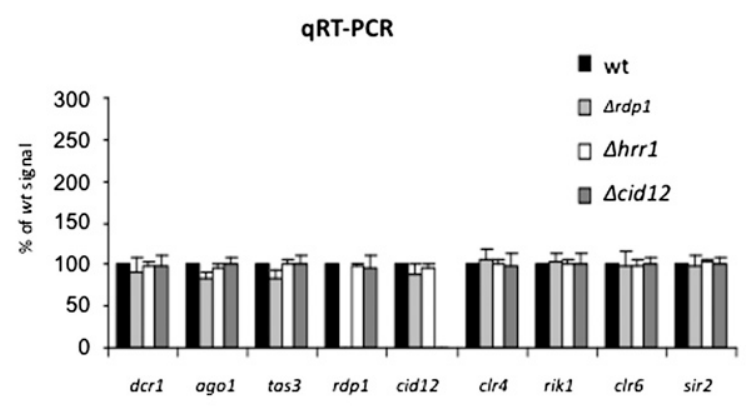

C

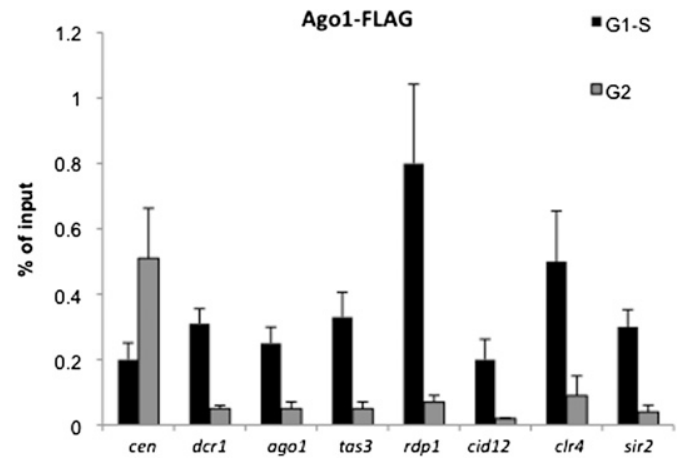

Figure 3. Autoregulation of RNAi genes. (A) Steady-state RNA analysis of RNAi genes in RNAi mutants. Total RNA was extracted from RNAi mutant strains, grown in minimal medium, blocked in G1-S, and measured by qRT-PCR using oligo dT. (B) Steady-state RNA analysis of RNAi genes in RDRC mutants, as in $A$. (C) Agol preferentially localizes to cen in G2 and to CGs in G1-S, but not to TGs (Supplemental Fig. 2C). ChIP analysis used anti-Flag in ago1-Flag-tagged strain blocked in G1-S or G2. Probes in the cen $d g$ region and several RNAi CGs were analyzed.

orientation of these RNAi genes is a defining feature. To test whether changing gene orientation from convergent to tandem leads to a different expression pattern, we introduced ura 4 between the key RNAi CGs (ago1-mmi1, spn6-dcr1, and meu6-clr4) and the non-RNAi nmt2-avn2 CG pair as a control, all in a $\Delta$ ura 4 strain. This effectively changes ago1, dcr1, clr4, and nmt2 from convergent to tandem orientation. We refer to these reoriented genes as 
"tandemized." The mutant strains so generated are called Tago1, Tdcr1, Tclr4, and Tnmt2. In these strains, the integrated ura4 is tandem with each RNAi gene, but in a convergent orientation with respect to downstream mmi1, spn6, meu6, and avn2, respectively (Fig. 4A-D). In these gene reconstructions, we maintained full promoter and terminator elements in ura4 (Birse et al. 1997). We also positioned ura4 between each CG so as to maintain promoter and terminator elements. Wild-type and tandemized mutant strains were blocked by HU treatment in G1-S and then released into G2. RT-PCR analysis systematically revealed that tandemized ago1, $d c r 1, c l r 4$, and nmt2 lose their transcriptional downregulation in G1-S, seen in wild type, so that their expression profile now remains constant during the cell cycle. Semiquantitative gel analysis of the RT-PCR products obtained is shown for all four tandemized gene arrangements (levels of DNA product are shown below each gel lane in Fig. 4A-D). Also, for tandemized Tago1 and Tdcr1, quantitative real-time RT-PCR was performed (Fig. 4A,B, right graphs), reaffirming the loss of G1-S down-regulation, as observed in wild type. We also examined protein levels of Ago1 in wild-type and Tago1 cells. Consistent with the transcript analysis, a significant decrease in Agol levels was observed in wild-type G1-S as compared with G2. Levels of Ago1, significantly, were both constant and twofold-elevated in G1-S and G2 Tago1 (Fig. 4A). In contrast, ura4, which is a TG in wild type, showed a significant decrease of mRNA levels in G1-S, as it is now repositioned as a CG. The other CGs-mmi1, spn6, meu6, and avn2-continued to display down-regulation in G1-S, since they remain in a

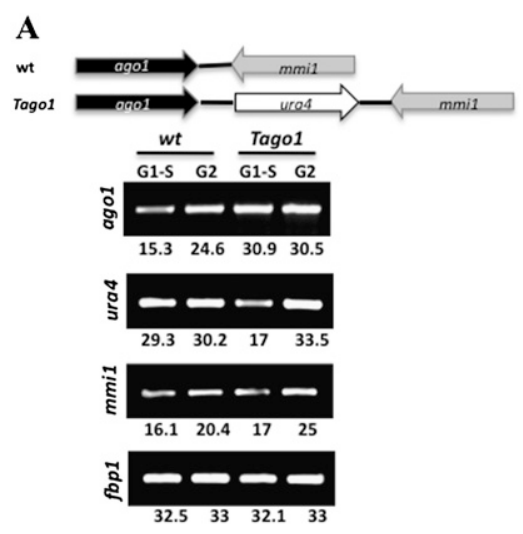

\section{B}
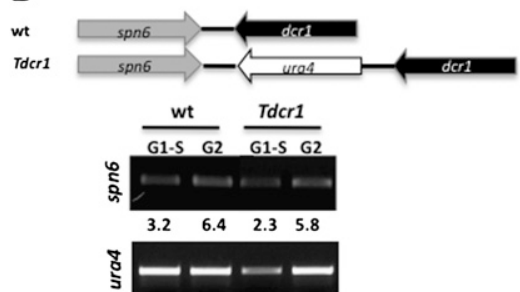

$\begin{array}{llll}24 & 23.6 & 15.4 & 23\end{array}$

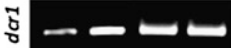
$\begin{array}{llll}16.6 & 30.8 & 31.9 & 32\end{array}$

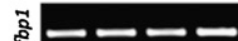

$\begin{array}{llll}19.6 & 21 & 21.5 & 22.1\end{array}$

C

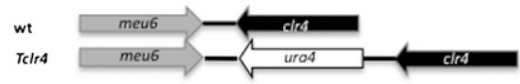

$\frac{w t}{G 1-S \quad G 2} \frac{T C l r 4}{G 1-S \quad G 2}$

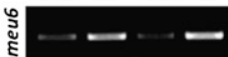

$\begin{array}{llll}2.9 & 8.3 & 2.1 & 9.2\end{array}$

亏

$\begin{array}{llll}25 & 25.7 & 17.2 & 27\end{array}$

는

$\begin{array}{llll}14.2 & 18.2 & 19.3 & 20\end{array}$

范 $=$

$\begin{array}{llll}18.8 & 19.2 & 19.6 & 18.1\end{array}$
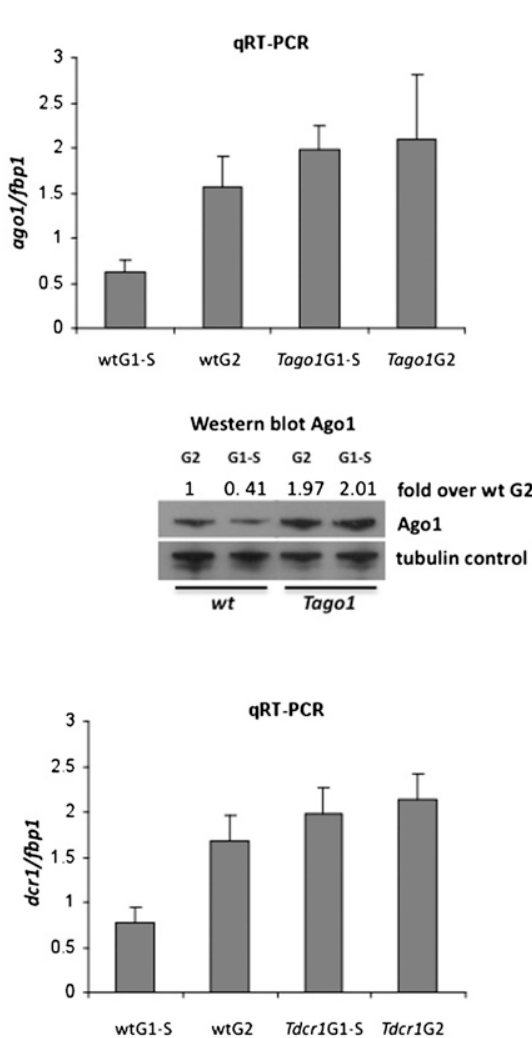

D

wet

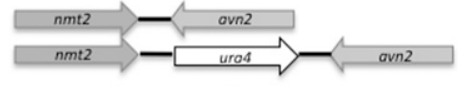

$$
\begin{aligned}
& \frac{w t}{G 1-S \quad G 2} \frac{\text { Tnmt2 }}{\text { G1-S G2 }} \\
& \text { ถั } \\
& \begin{array}{llll}
21 & 31 & 32.3 & 32.5
\end{array} \\
& \text { ป } \longrightarrow \\
& \begin{array}{lllll}
24.3 & 25 & 18.5 & 28.9
\end{array} \\
& \begin{array}{lllll}
\text { ₹ิ } & & & & \\
\hline & & & & \\
\hline & 2.3 & 5.8 & 2.1 & 6.2
\end{array} \\
& \text { है } \begin{array}{lllll}
32 & 31.2 & 31 & 30.7
\end{array}
\end{aligned}
$$

Figure 4. Gene orientation dictates RNAi CG expression during the cell cycle. $(A-D)$ ura4 was introduced between the four CGs by homologous recombination as indicated, changing the orientation of adjacent RNAi CGs to tandem and itself becoming a CG. Total RNA was isolated from wild type and tandem mutants and blocked in G1-S by $\mathrm{HU}$ or released into G2, and RNA levels were determined by RT-PCR. Quantification shown below bands is expressed in arbitrary units. Multiple repeats of these experiments gave similar values within a $5 \%$ range. Specific RT primers were positioned within ORFs. Tandem $f b p 1$ was used as a control. ( $A, B$, right graphs) Tago1 and Tdcr1 were further quantitated by real-time RT-PCR. Also shown in $A$ is a Western blot analysis of Agol in wild-type or Tago1 cells blocked in G1-S by HU or released to G2. Anti-Agol antibody was used to detect Agol protein levels. Equal levels of total protein extracts were confirmed by Western blotting using anti-tubulin antibody. 
convergent direction to ura4 (Fig. 4A-D). These results confirm that convergent orientation affects gene expression during cell cycle, and furthermore suggest that the convergent orientation for RNAi genes may relate to their function during the cell cycle.

We showed previously that CGs generate readthrough mRNAs in G1-S phase. We wished to test whether orientation switching affects this readthrough profile. 3'RACE analysis of wild-type ago1 and dcr1 genes in G1-S revealed the expected readthrough bands (Fig. 5A). These are extended mRNAs derived from Pol II transcription that fails to use the proximal polyA site, instead reading through to more distal polyA signals. In marked contrast, a single band, corresponding to mRNA terminated after the proximal polyA site, was observed in G1-S for both tandem-oriented ago1 and dcr1 (Tago1 and Tdcr1 mutants). Notably, this confirms that the ura4 insertion in Tago1 and Tdcr1 has not interrupted ago1 or dcr1
polyA signals. Effectively, the switch from proximal polyA site in G2 to readthrough transcripts in G1-S is lost in the tandem-oriented ago1 and dcr1 mutants (Fig. 5A). Consistent with the loss of dsRNA in Tago1, Tdcr1, and Tclr4, we also observed a loss of G1-S-specific heterochromatin marks over these modified gene loci (Fig. $5 B)$. In view of the clear difference in heterochromatin status between tandemized and wild-type convergent RNAi genes, we searched for the presence of ago1 siRNAs in G1-S-phase wild-type cells and their absence in Tago1 cells. To maximize the sensitivity of this experiment, we used an improved Northern blot technique using a chemical (EDC) cross-linking procedure that favors small RNA detection (Pall et al. 2007). Furthermore, G1-S cells were selected for by nitrogen starvation (Fig. 5C, left, FACS) and the siRNA fraction enriched by polyethylene glycol 8000 precipitation (see the Materials and Methods for details). Note that nitrogen starvation blocks cells in late
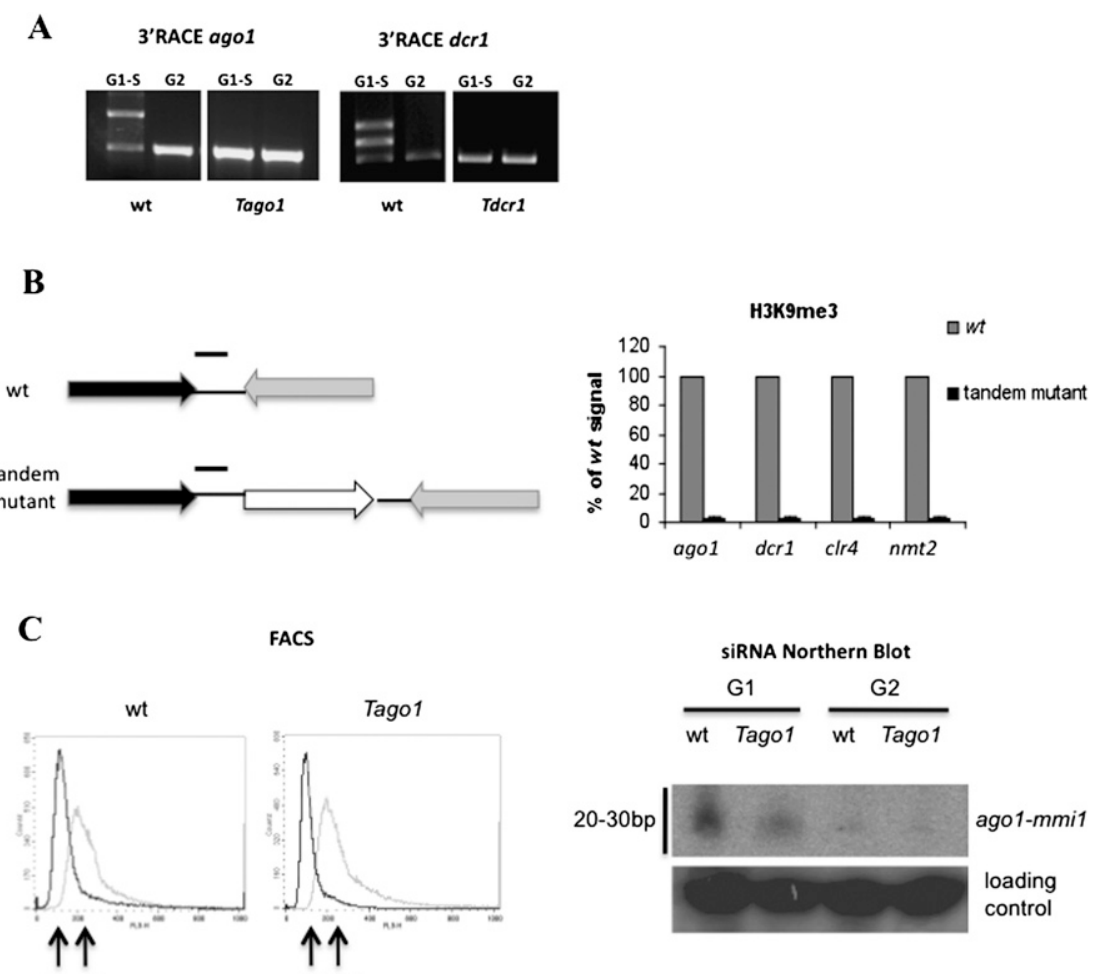

FACS

$$
1 \mathrm{~N} 2 \mathrm{~N}
$$

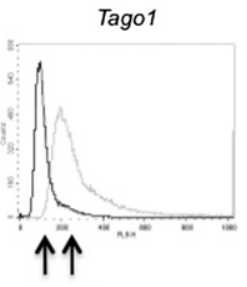

$1 \mathrm{~N} 2 \mathrm{~N}$

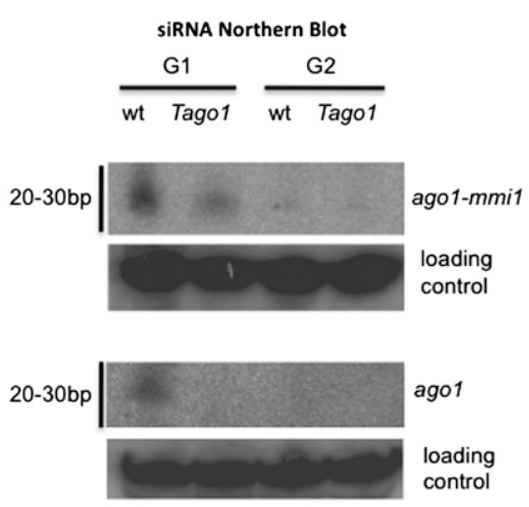

Figure 5. Tandemized CGs lose G1-S-specific RNAi-mediated heterochromatin. $(A)$ 3'RACE analysis of ago1 and dcr1 using RNA isolated from wild-type, Tago1, or Tdcr1 cells blocked in G1-S by HU or released to G2. Phased oligo dT was used for reverse transcription. DNA was amplified by PCR using phased dT and specific forward primer within ago1 or dcr1 ORFs. (B) ChIP analysis using H3K9me3 antibody over intergenic region in wild-type or tandemized RNAi genes, as indicated in the left gene maps. Black bar denotes position of PCR primer amplicon. Graphs on the right show qPCR ChIP signals as indicated. $(C)$ Northern blot analysis of ago1-mmi1 CG siRNAs from G1 and G2 cells. (Left) FACs analysis of G1-selected cells using nitrogen starvation (dark trace) or unsynchronized predominantly G2 cells (light trace). G1 cells are predominantly haploid, while other cells were diploid. (Right) Northern blot of ago1-mmi1 siRNAs or ago1 alone siRNAs isolated from G1 or G2 enriched cells. Loading control is tRNA detected by nonspecific hybridization to oligonucleotide probes and indicates near-equal loading of small RNA samples to each lane. Quantification is presented below the Northern blot analysis, showing the average values obtained from three independent experiments.
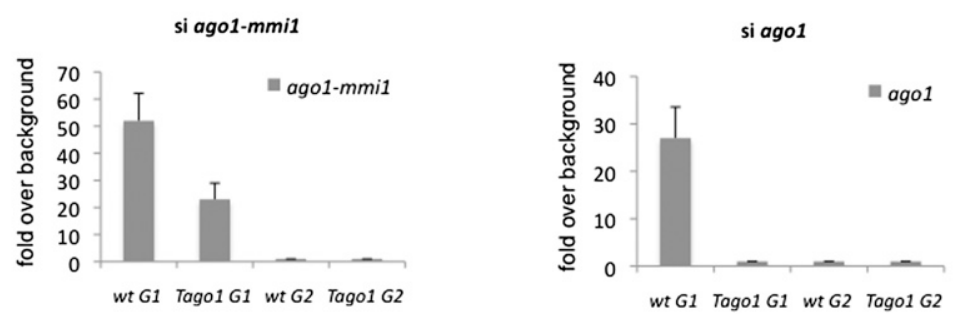
G1 (Sabatinos and Forsburg 2010) rather than the HUinduced S-phase block. This slightly early block in the cell cycle may favor higher siRNA accumulation. With ${ }^{32}$ P-labeled oligo probes across ago1-mmi1, siRNA was detected in G1, but not significantly in G2 cells. Note that G1 Tago1 cells still produce mmi1 siRNAs, as this gene is now convergent with ura4. Importantly, with solely ago1 oligo probes, siRNA was detected in G1 wildtype but not Tago1 cells (Fig. 5C). These crucial experiments were repeated multiple times, and their quantification is presented below selected Northern blots in Figure 5C.

Overall, these results demonstrate that the CG status of RNAi genes is required for down-regulation in G1-S at both mRNA and protein levels. This is clearly demonstrated by switching their orientation from convergent to tandem. Even though several-hundred nucleotides of $5^{\prime}$ promoter and $3^{\prime}$ terminator sequences were preserved on either side of the tandemized RNAi genes, we found that switching gene orientation was dominant over potential local cis-acting elements in preventing G1-S readthrough transcription. Consequently, heterochromatin marks were lost and transcription was no longer G1-S down-regulated. These results provide an unanticipated example of how gene orientation can affect gene expression.

\section{Overexpression of agol and dcrl causes aberrant centromeric transcription and gene silencing}

We sought to establish the biological function for cell cycle-dependent down-regulation of RNAi genes. A potentially related observation is that increased levels of centromeric transcription (both sense and antisense) occur in G2 with dcr1 and clr4 deletion mutants, but only a slight increase in swi6 mutant cells (Motamedi et al. 2008). Notably, swi6 is a TG. We therefore examined whether altered expression levels of ago1 and dcr1 could affect centromeric transcription. We compared our tandemized strains that display increased gene expression in G1-S phase with strains either deleted for ago1 or dcr1 or overexpressing ago1 and dcr1 from transformed plasmids (Buker et al. 2007; Colmenares et al. 2007).

RT-PCR analysis of RNA isolated from wild-type, Tago1, Tdcr1, Tclr4, and Tnmt2 cells blocked in G1-S revealed that the levels of centromeric antisense $d g$ transcripts were significantly reduced in Tago1 and Tdcr 1 cells. With Tclr4 cells, the effect on centromeric transcription was only slight, while the control Tnmt2 cells showed no effect (Fig. 6A). Pol II levels at centromeres in wild-type, Tago1, Tdcr1, Tclr4, and Tnmt2 cells blocked in G1-S were also tested. Pol II occupancy over the centromere ( $d g$ region) correlated with the dg RT-PCR analysis, revealing significant reduction in Tago1 and Tdcr1 cells but only a mild (20\%) reduction in Tclr4 cells. Tnmt2 again had no effect on Pol II at centromeres (Fig. 6B). TG $f b p 1$ was used as a control and showed no significant change in Pol II occupancy for the different strains. We next carried out RT-PCR analysis on $d g$ antisense RNA from $\Delta a g o 1, \Delta d c r 1,+a g o 1$, and $+d c r 1$ strains, all blocked in G1-S, and observed a significant reduction of $d g$ transcripts in cells overexpressing ago1 or dcr1. In contrast, ago1 or dcr1 deletion caused the accumulation of centromeric transcripts (Fig. 6C). Decreased levels of centromeric transcripts in cells overexpressing ago1 or dcr1 could be due to either posttranscriptional processing or lower Pol II levels. Therefore, we performed Pol II chromatin immunoprecipitation (ChIP) experiment on wild-type, +ago1, and $+d c r 1$ cells and observed that Pol II levels were reduced in $+a g o 1$ or $+d c r 1$ cells. The TG $f b p 1$ was used as a control and showed no change in Pol II occupation (Fig. 6D). To further investigate cen transcription in Tago1 cells, we finally quantitated the levels of cen siRNA in G1-S and G2. Interestingly, in contrast to Pol II-derived cen transcription, which decreased in Tagol cells, siRNA levels increase by about twofold (Fig. 6E). We predict that higher Agol levels in Tago1 cells may act to stabilize siRNA, resulting in higher siRNA detection.

A clear biological measure of altered centromeric transcription is the degree of reporter gene silencing when inserted within the centromeric repeats. We therefore used a strain with ade6 positioned at the right repeat of cen1 (Allshire et al. 1994). Cells were grown on EMM plates with low adenine. Silencing at centromeres in wild-type cells lead to pink colony formation. Impaired silencing in $\Delta a g o 1$ lead to active transcription of ade6 and, consequently, white colonies. Cells overexpressing ago1 showed a dark-red color, suggesting even more stable silencing at centromeres compared with wild-type cells. Tago1 colonies were dark pink, an intermediate phenotype (Fig. 6F). This observation correlates with the fact that, in Tago1 cells, Agol levels are increased compared with wild type, but are lower than in +ago1 cells. We finally tested ade6 centromeric silencing for the other tandemized mutants. Like Tago1, Tdcr1 and Tclr4 gave the same intermediate silencing phenotype, while the control Tnmt2 strain gave a wild-type silencing phenotype.

\section{Mutant strains with tandemized RNAi genes display aberrant cellular phenotypes}

To look for cytological phenotypes, we examined wildtype, Tago1, Tdcr1, Tclr4, and Tnmt2 cells by light microscopy. Notably, Tago1 and Tdcr1 cells were visibly smaller than wild-type and Tnmt2 cells. Tclr4 cells were thicker, but otherwise similar to wild-type cells (Fig. 7A). These altered cell morphologies indicate significant cell growth defects. Next, based on a previous study (Carmichael et al. 2004), we used DAPI staining to visualize mitotic nuclei. Tago1, Tdcr1, and Tclr4 cells displayed a high incidence of aberrantly extended chromosomes compared with wild type and Tnmt2 (Fig. 7B). This phenotype, known as "archery bow," indicates that, when cells enter mitosis, centromeres separate, but the distal regions of chromosomes remain associated (Funabiki et al. 1993, 1996), causing a delay in separation. This phenotype suggests that the cell cycle regulation of RNAi genes (lost in the tandem constructs) is linked directly or indirectly to cohesin and condensin function. We also examined the $+a g o 1$ and $+d c r 1$ cells by light microscopy and observed that a majority of cells were smaller and had 
A

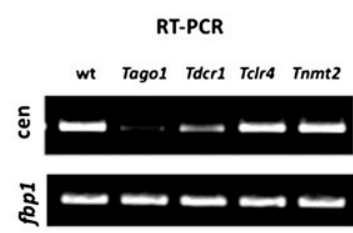

C

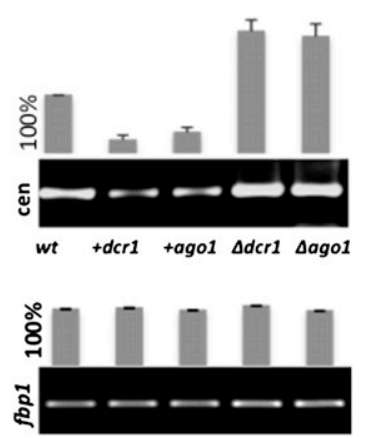

E

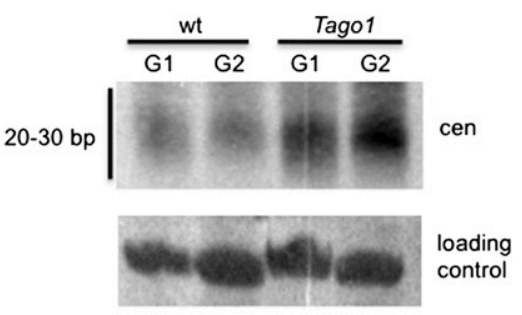

B

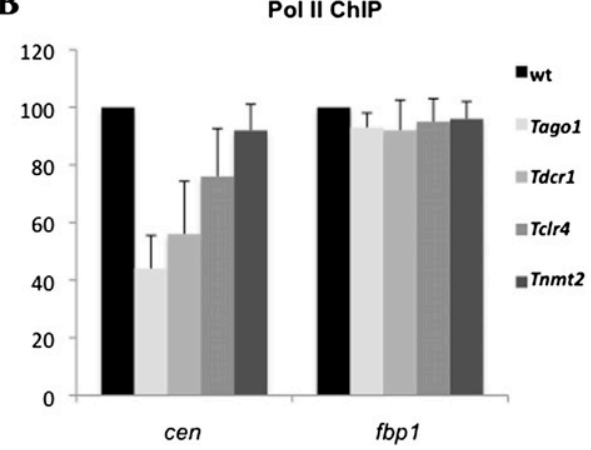

D

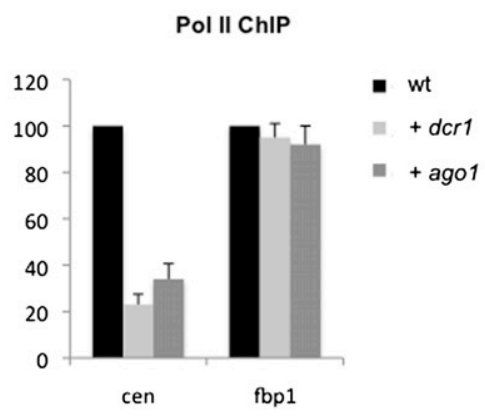

F

Silencing assay

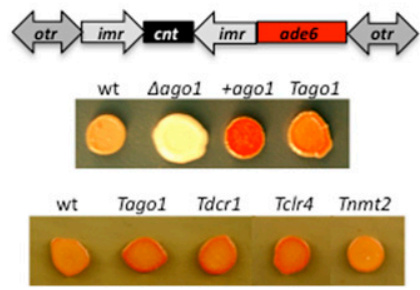

Figure 6. Altered ago1 and $d c r 1$ expression inhibits centromeric transcription and causes aberrant gene silencing. $(A)$ RT-PCR analysis of RNA isolated from wild-type, Tago1, Tdcr1, Tclr4, and Tnmt2 strains. RT primers within $d g$ and ORFs of ago1-, dcr1-, clr4-, and $f b p 1$-generated cDNAs were amplified by PCR. (B) Pol II ChIP signals are reduced at cen in chromatin isolated from Tago1, Tdcr1, and Tclr4 cells. Tnmt2 does not affect Pol II occupancy at cen. Tandem fbp1 gene was used as control, showing similar Pol II levels in all tested strains. (C) RT-PCR analysis showing effect of RNAi gene dosage (either overexpression or gene deletion) on cen and CG transcription. RT-PCR products were analyzed on agarose gel and quantified using Mass Quant software. Signals were compared with wild-type signal (set as $100 \%$ ). Error bars are based on three biological repeats. $(D)$ Pol II reduction at cen in cells overexpressing ago1 or $d c r 1$. TG $f b p 1$ was used as a control. Pol II ChIP signals were compared with wildtype signal, which was set as $100 \%$. $(E)$ Northern blot analysis of cen siRNAs from G1-S or G2 wild-type or Tago1 cells as in Figure 5C. (F) Silencing assay using ade6 marker integrated at cnt1, as depicted. Reporter strain is denoted as wild type. Aago1, +ago1, and Tago1 have the same reporter background. Cells were grown on EMM-low ade plates. Pink corresponds to silenced ade6 marker. White in $\Delta a g o 1$ cells reflects impaired silencing at centromeres. Overexpression of ago1 causes dark-red colonies. Tandem ago1 shows an intermediate red. abnormal morphological phenotypes (Fig. 7C) and higher proliferation (data not shown), similar to previous studies (Carmichael et al. 2004). These phenotypes may be connected to ago1 and dcr1 function in cell cycle regulation rather than defects at centromeric transcription (Carmichael et al. 2004), or possibly a combination of both.

Overall, these phenotypic data show that overexpression of ago1 and dcr1, by either tandemizing or transformation of expression plasmids, affects centromeric transcription in G1-S. This leads to both gene silencing and cytological defects that may impact on cell cycle progression.

\section{Discussion}

We describe different features of cen and CG heterochromatin. First, the RDRC complex, although essential for cen heterochromatin formation, is not required for CG heterochromatin. The transient nature of CG heterochromatin suggests that Pol II sense and antisense transcription is sufficient to generate dsRNA for CGs without relying on Rdp1 amplification. In contrast, cen heterochromatin is a more permanent state that requires RDRC amplification of centromeric transcripts. How RDRC is recruited to centromeres but not CGs remains to be established. Importantly, we now provide direct evidence for the formation of siRNA from CG dsRNA. Thus, both Dcrl and Agol RNA processing activities are required for CG heterochromatin formation (Fig. 2E,F). Furthermore, low levels of siRNA from ago1-mmi1 CGs are detectible using an improved Northern blot procedure and, appropriately, G1 synchronized cells (Fig. 5C). Significantly, these siRNAs are only detected in G1 phase but not from ago1 when it is in tandem orientation, as in Tago1. 
A
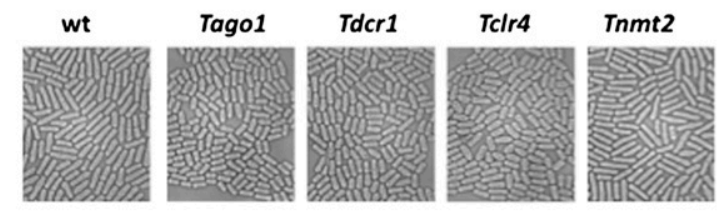

B
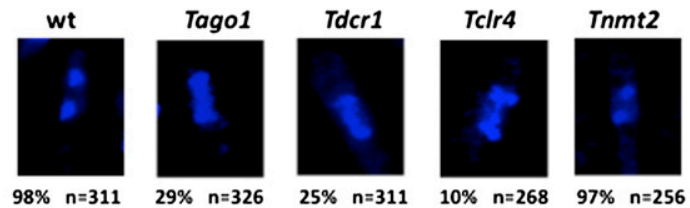

C

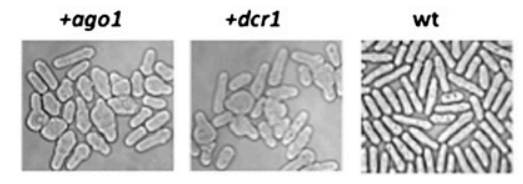

D

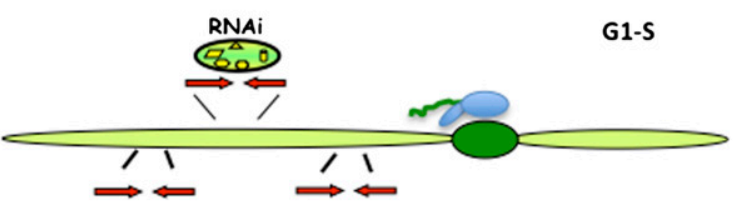

G2

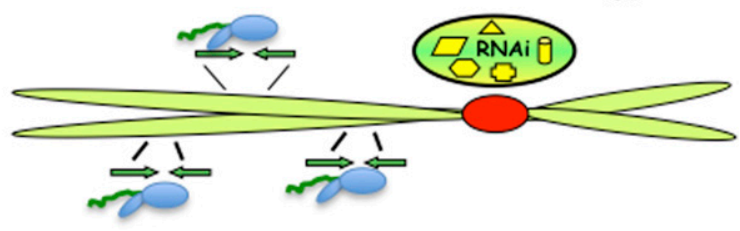

Figure 7. Strains harboring tandem-oriented RNAi genes show defective cellular phenotypes. (A) Wild-type, Tago1, Tdcr1, Tclr4, and Tnmt2 cells were observed by light microscopy. Exponential cultures were grown in EMM medium. (B) Analysis of DAPI-stained mitotic nuclei in wild-type, Tago1, Tdcr1, Tclr4, and Tnmt2 cells reveals archery bow phenotype in Tago1, Tdcr1, and Tclr4 cells. Values below selected cells show percentage of cells with the shown phenotype, based on indicated sample numbers. Wild type and Tnmt2 show the expected wildtype pattern, while Tago1, Tdcr1, and Tclr4 show the mutant phenotype. $(C)+a g o 1$ and $+d c r 1$ have aberrant morphology compared with wild type. Cells were grown in EMM medium and analyzed by light microscopy using a $100 \times$ objective. $(D)$ Model of cell cycle-dependent RNAi regulation of heterochromatin. Transcription of cen repeats is detectable in G1-S, when CGs are silenced by RNAi-dependent formation of transient heterochromatin. CGs are highly transcribed in G2, when cen is silenced. RNAi apparatus is denoted as ovals, while elongating Pol II is indicated in cartoon format. Red arrows denote silenced RNAi CGs in G1-S, while green arrows denote active RNAi CGs in $\mathrm{G} 2$.
We demonstrate that RNAi genes are convergent and down-regulated in G1-S, implying a biological purpose for this gene arrangement. An exception to this rule is the CG clr6. However, Clr6 exists in two complexes with Sin 3 and is essential for viability (Nicolas et al. 2007) and regulation of the checkpoint kinase Cds1 (Kunoh et al. 2008). This suggests a more complex gene regulatory process. The key importance of RNAi CG arrangement is underlined by our analysis of tandem ago1 and dcr1 genes (Tago1 and Tdcr1). The deregulation of their expression, when switched to a tandem orientation, shows clear defects in cell division and morphology. This provides a striking paradigm for the importance of gene position along a chromosome.

Faithful chromosome segregation is essential for successful completion of the cell cycle. RNAi mutants are known to display frequent defects, such as lagging chromosomes or chromosome loss during mitosis. This is related to incorrect sister chromatid orientation and the defective attachment of the kinetochore to the spindle (Provost et al. 2002; Hall et al. 2003). These data support a model in which chromosome architecture is regulated by the RNAi pathway. Furthermore, RNAi core proteins have been shown to perform functions related to cell cycle regulation (Carmichael et al. 2004). In fission yeast, Agol and Dcrl regulate hyperphosphorylation of the mitotic regulator $\mathrm{Cdc} 2$ upon genotoxic insult. Under normal conditions, DNA damage activates signaling pathways, which leads to inhibitory phosphorylation of Cdc2 and cell cycle arrest (Rhind et al. 1997; Rhind and Russell 1998). Once DNA damage is corrected, Cdc2 is dephosphorylated by Cdc 25 mitotic phosphatase and cell cycle is restored (Russell and Nurse 1986; Millar et al. 1991). ago1- and dcr1-null mutants fail to block mitosis in response to DNA damage, most probably due to the lack of Cdc2 hyperphosphorylation. These mutant cells divide unchecked, and so result in unequal chromosome divisions. Surprisingly, overexpression of human ago1 rescues checkpoint deficiencies in the dcr1-null strain (Carmichael et al. 2004), suggesting that Agol in higher eukaryotes also plays a role in cell cycle regulation. In addition, it has been shown that Agol binds to 14-3-3 proteins that function in cell cycle regulation. Also, overexpression of the Agol $\mathrm{N}$ terminus leads to cell cycle delays through inhibition of the nuclear import of cyclin kinase Cdc25 (Stoica et al. 2006).

As shown in these studies, overexpression of ago1 or dcr1 show striking morphological phenotypes. Cells lose their elongated shape and display hyperproliferation. This phenotype has been observed previously (Carmichael et al. 2004). A milder version of the +ago1 and $+d c r 1$ phenotypes is clearly visible in Tago1 and Tdcr1, suggesting that the dosage of these proteins must be tightly regulated. The intermediate phenotype of Tclr4 is consistent with the position of Clr4 in the RNAi pathway. Thus, higher levels of Dcrl and Agol show strong phenotypes, as they act upstream in heterochromatin establishment. Indeed, high levels of RNAi core proteins may be cytotoxic. Notably, elevated levels of human Ago2 have been observed in a breast cancer cell line, where 
ago2 overexpression enhances cell proliferation and reduces cell-cell adhesion (Adams et al. 2009). In addition, the Burkitt's lymphoma-derived cell lines express fourfold higher levels of Dicer mRNA than normal human lymphocytes (Hecht and Aster 2000). Clearly, core RNAi proteins play a role in signaling and cell cycle regulation.

We propose a general model for cell cycle regulation in $S$. pombe (Fig. 7D) that may resolve the paradox of a transcriptional requirement to silence centromeric transcription. During mitosis, H3K9me and Swi6 occupancy on centromeric chromatin is altered by phosphorylated H3S10, which correlates with condensin recruitment (Hirano 2005). Cut3, a condensin subunit, displays enrichment in mitosis, but delocalizes from centromeres in G1-S (Chen et al. 2008). This opens up a short window of time for centromeric transcription in G1-S. We suggest that the RNAi machinery, precisely at this time period, preferentially acts on CGs, occurring at multiple positions along the chromosomal arms, where it leads to their down-regulation. This effective sequestration of the RNAi machinery to CGs destabilizes CEN heterochromatin, allowing its unrestricted transcription. Consistent with our model, deregulated levels of Agol or Dcr1 lead to a decrease in cen G1-S transcription (Fig. 6). RNAi gene expression is also down-regulated during G1-S through RNAi-mediated heterochromatin formation. Cohesin recruitment then blocks transcriptional readthrough in G2, and so prevents dsRNA formation (Gullerova and Proudfoot 2008). This results in translatable mRNAs and RNAi protein production in G2. Centromeric RNAs formed during $S$ phase are quickly processed by RNAi and lead to heterochromatin restoration until the next $M$ phase. Overall, our results demonstrate an autoregulatory process for RNAi-induced heterochromatin that neatly perpetuates these epigenetic marks through multiple rounds of cell division.

\section{Materials and methods}

\section{Yeast strains}

S. pombe $972 h^{-}$was used in this study as wild type. Growth conditions and all genetic manipulations were carried out as described previously (Moreno et al. 1991). The S. pombe strains used in this study are summarized in Supplemental Table 1.

\section{Silencing assay}

The $S$. pombe strain with a reporter adeb gene inserted in centromere was used as wild type for the silencing assay. Exponentially growing cells were serially diluted and dropped onto EMM-low ade plates. Growth of cells was checked on EMM complete plates. Chromosome instability was tested on YES containing $10 \mathrm{ug} / \mathrm{mL}$ thiabendazol plates.

\section{ChIP}

See the Supplemental Material for ChIP. Cells used are shown in Supplemental Table 1, antibodies are shown in Supplemental Table 2, and oligonucleotide primers are shown in Supplemental Table 3.

\section{Cell cycle arrest}

HU was used to block cells in early $S$ phase. Exponential cell culture was treated by $11 \mathrm{mM} \mathrm{HU}$ for $4 \mathrm{~h}$. Cells were washed twice with $50 \mathrm{~mL}$ of water and released for $1 \mathrm{~h}$ to grow. Cycling cells were used to provide predominantly G2 cell cycle chromatin $(90 \%)$. Cdc10ts cells were blocked in G1 by a temperature shift $\left(37^{\circ} \mathrm{C}\right)$. Cells were stained by propidium iodide and checked by FACS.

\section{Northern blotting of siRNAs}

Wild-type and Tago1 cells were grown in EMM medium for $20 \mathrm{~h}$, washed, split into EMM and EMM-N, and incubated overnight at $25^{\circ} \mathrm{C}$. Cell phase of the cultures was confirmed by FACS. RNA was extracted by resuspending cells in $50 \mathrm{mM}$ Tris- $\mathrm{HCl}(\mathrm{pH} 7.5)$, $10 \mathrm{mM}$ EDTA (pH 8), $100 \mathrm{mM} \mathrm{NaCl}$, and 1\% SDS. Acid phenolchloroform and acid-washed glass beads were added, followed by three cycles of homogenization. Soluble fraction was extracted with isopropanol. siRNA were precipitated as follows: RNA pellet was dissolved in $300 \mu \mathrm{L}$ of TE buffer with addition of equal amounts of PEG solution (20\% PEG 8000, $2 \mathrm{M} \mathrm{NaCl}$ ). Samples were mixed and incubated for at least $30 \mathrm{~min}$ on ice, followed by centrifugation at $14,000 \mathrm{~g}$ for $15 \mathrm{~min}$. siRNAs were precipitated from supernatant by isopropanol.

Concentration of siRNA was measured by nano Drop. Sixty micrograms of total siRNA were loaded and separated on $17.5 \%$ polyacrylamide/7 M urea gel, followed by transfer onto positively charged nylon membrane (Roche). Cross-linking was performed as follows: For $320-\mathrm{cm}^{2}$ membrane, $122.5 \mu \mathrm{L}$ of 12.5 M 1-methylmidazol was added to $10 \mathrm{~mL}$ of DEPC-treated water and $\mathrm{pH}$ was adjusted to $8 \mathrm{by} \mathrm{HCl}$. This was used to dissolve 0.373 $\mu \mathrm{g}$ of $\mathrm{N}$-(3-dimethylaminopropyl)-N-ethylcarbodiimide (EDC). Whatman paper (membrane size) was saturated with EDC solution. Membrane with the RNA side on top was placed on the EDC-saturated Whatman paper, wrapped in cling film, and incubated for $2 \mathrm{~h}$ at $65^{\circ} \mathrm{C}$. Cross-linked membranes were prehybridized with ULTRAhyb-Oligo Hybridization buffer (Ambion) for at least $30 \mathrm{~min}$.

Mixtures of primers complementary to cen, ago1, or mmi1 were labeled with T4 PNK and $20 \mathrm{pmol}^{32} \mathrm{P}-\gamma$-ATP. Probes were incubated with membranes overnight at $35^{\circ} \mathrm{C}$. Membranes were washed with $2 \times$ SSC and $0.5 \%$ SDS for $1 \mathrm{~h}$ and visualized using a PhosphorImager. Oligonucleotides used as probes were as follows: cen: dg1, CTACTCTTCTCGATGATCCTG; dg2, GTA GTACGACGATGATGTGTTTC; dh1, ATTCTTTCTGAACCT CTCTGTTAT; dh2, TTTGATGCCCATCTTCATTCCACTTG; dh3, GGGCGTACATCATTCCTACTTCGATA; dh4, TACTGT CATTAGGATATGCTCA. ago1-mmi1: ago1-1, TATTAGAGGG AGTGTTGAA; ago1-2, CCAGTATCATGTAAGTTCC; ago1-3, TAACGTAGAATTGCATCAAA; ago1-4, AGAGATATACTGC AAAACTG; ago1-5, ATACCCAAATCCAACCCTTT; ago1-6, CACCCATCTCTGCAAGGCGT; ago1-7, AGCCGATGATACT TTTGTTG; ago1-8, ACAAAGATGTGGTATATGTA; ago1-9, TGTTTTTATTTTGCTTTATA; ago1-10, TTCAAATTTGAAA ACAGCCT; mmi1-1, CTCCTATCGGTAGAAGATTG; mmi1-2, ACGAGAACGGCTATCATAGT; mmi1-3, AGCATCCAACAT TCTTGAAA; mmi1-4, AATCCAGCAAAAGGGAGATA.

\section{Other RNA and protein isolation and analysis}

See the Supplemental Material for other methods of RNA and protein analysis.

\section{Microscopy}

Cells were grown to mid-log phase, collected by centrifugation $(1000 \mathrm{~g})$ for $3 \mathrm{~min}$, washed with water, and dropped onto slides. 
Cells were fixed by boiling at $65^{\circ} \mathrm{C}$ and stained with DAPI in $50 \%$ glycerol solution.

\section{Acknowledgments}

We thank the Moazed laboratory for support and technical advice, especially Erica Gerace. We also thank Thomas Gligoris for valuable advice and comments. We gratefully received strains from Robin Allshire, Shiv Grewal, Chris Norbury, and Yoshi Watanabe. Part of these studies was performed in the Moazed laboratory at Harvard Medical School, funded by an EMBO shortterm fellowship to M.G. This work was supported by a Wellcome Trust program and a Cancer Research UK project grant to N.J.P. and HHMI to D.M.

\section{References}

Adams BD, Claffey KP, White BA. 2009. Argonaute-2 expression is regulated by epidermal growth factor receptor and mitogen-activated protein kinase signaling and correlates with a transformed phenotype in breast cancer cells. Endocrinology 150: 14-23.

Allshire RC, Javerzat JP, Redhead NJ, Cranston G. 1994. Position effect variegation at fission yeast centromeres. Cell 76: 157-169.

Aranda A, Proudfoot NJ. 1999. Definition of transcriptional pause elements in fission yeast. Mol Cell Biol 19: 12511261.

Birse CE, Lee BA, Hansen K, Proudfoot NJ. 1997. Transcriptional termination signals for RNA polymerase II in fission yeast. EMBO J 16: 3633-3643.

Buhler M, Spies N, Bartel DP, Moazed D. 2008. TRAMPmediated RNA surveillance prevents spurious entry of RNAs into the Schizosaccharomyces pombe siRNA pathway. Nat Struct Mol Biol 15: 1015-1023.

Buker SM, Iida T, Buhler M, Villen J, Gygi SP, Nakayama J, Moazed D. 2007. Two different Argonaute complexes are required for siRNA generation and heterochromatin assembly in fission yeast. Nat Struct Mol Biol 14: 200-207.

Carmichael JB, Provost P, Ekwall K, Hobman TC. 2004. agol and dcrl, two core components of the RNA interference pathway, functionally diverge from rdp1 in regulating cell cycle events in Schizosaccharomyces pombe. Mol Biol Cell 15: $1425-1435$.

Carthew RW, Sontheimer EJ. 2009. Origins and mechanisms of miRNAs and siRNAs. Cell 136: 642-655.

Chen ES, Zhang K, Nicolas E, Cam HP, Zofall M, Grewal SI. 2008. Cell cycle control of centromeric repeat transcription and heterochromatin assembly. Nature 451: 734-737.

Colmenares SU, Buker SM, Buhler M, Dlakic M, Moazed D. 2007. Coupling of double-stranded RNA synthesis and siRNA generation in fission yeast RNAi. Mol Cell 27: 449-461.

Czech B, Malone CD, Zhou R, Stark A, Schlingeheyde C, Dus M, Perrimon N, Kellis M, Wohlschlegel JA, Sachidanandam $\mathrm{R}$, et al. 2008. An endogenous small interfering RNA pathway in Drosophila. Nature 453: 798-802.

Funabiki H, Hagan I, Uzawa S, Yanagida M. 1993. Cell cycledependent specific positioning and clustering of centromeres and telomeres in fission yeast. J Cell Biol 121: 961-976.

Funabiki H, Kumada K, Yanagida M. 1996. Fission yeast Cut1 and Cut2 are essential for sister chromatid separation, concentrate along the metaphase spindle and form large complexes. EMBO I 15: 6617-6628.

Grewal SI, Jia S. 2007. Heterochromatin revisited. Nat ReV Genet 8: 35-46.
Gromak N, West S, Proudfoot NJ. 2006. Pause sites promote transcriptional termination of mammalian RNA polymerase II. Mol Cell Biol 26: 3986-3996.

Gullerova M, Proudfoot NJ. 2008. Cohesin complex promotes transcriptional termination between convergent genes in $S$. pombe. Cell 132: 983-995.

Hall IM, Noma K, Grewal SI. 2003. RNA interference machinery regulates chromosome dynamics during mitosis and meiosis in fission yeast. Proc Natl Acad Sci 100: 193-198.

Hansen K, Birse CE, Proudfoot NJ. 1998. Nascent transcription from the nmt1 and nmt2 genes of Schizosaccharomyces pombe overlaps neighbouring genes. EMBO J 17: 3066-3077.

Hecht JL, Aster JC. 2000. Molecular biology of Burkitt's lymphoma. J Clin Oncol 18: 3707-3721.

Hirano T. 2005. Condensins: organizing and segregating the genome. Curr Biol 15: R265-R275. doi: 10.1016/j.cub.2005.03.037.

Irvine DV, Zaratiegui $\mathrm{M}$, Tolia NH, Goto DB, Chitwood DH, Vaughn MW, Joshua-Tor L, Martienssen RA. 2006. Argonaute slicing is required for heterochromatic silencing and spreading. Science 313: 1134-1137.

Kloc A, Martienssen R. 2008. RNAi, heterochromatin and the cell cycle. Trends Genet 24: 511-517.

Kloc A, Zaratiegui M, Nora E, Martienssen R. 2008. RNA interference guides histone modification during the $\mathrm{S}$ phase of chromosomal replication. Curr Biol 18: 490-495.

Kunoh T, Habu T, Matsumoto T. 2008. Involvement of fission yeast Clr6-HDAC in regulation of the checkpoint kinase Cds1. Nucleic Acids Res 36: 3311-3319.

Lengronne A, Katou Y, Mori S, Yokobayashi S, Kelly GP, Itoh T, Watanabe Y, Shirahige K, Uhlmann F. 2004. Cohesin relocation from sites of chromosomal loading to places of convergent transcription. Nature 430: 573-578.

Mattick JS. 2009. The genetic signatures of noncoding RNAs. PLoS Genet 5: e1000459. doi: 10.1371/journal.pgen.1000459.

Mayr C, Bartel DP. 2009. Widespread shortening of 3'UTRs by alternative cleavage and polyadenylation activates oncogenes in cancer cells. Cell 138: 673-684.

Millar JB, McGowan CH, Lenaers G, Jones R, Russell P. 1991. p80cdc25 mitotic inducer is the tyrosine phosphatase that activates $\mathrm{p} 34 \mathrm{cdc} 2$ kinase in fission yeast. EMBO J 10: 43014309.

Moreno S, Klar A, Nurse P. 1991. Molecular genetic analysis of fission yeast Schizosaccharomyces pombe. Methods Enzymol 194: 795-823.

Motamedi MR, Verdel A, Colmenares SU, Gerber SA, Gygi SP, Moazed D. 2004. Two RNAi complexes, RITS and RDRC, physically interact and localize to noncoding centromeric RNAs. Cell 119: 789-802.

Motamedi MR, Hong EJ, Li X, Gerber S, Denison C, Gygi S, Moazed D. 2008. HP1 proteins form distinct complexes and mediate heterochromatic gene silencing by nonoverlapping mechanisms. Mol Cell 32: 778-790.

Neil H, Malabat C, d'Aubenton-Carafa Y, Xu Z, Steinmetz LM, Jacquier A. 2009. Widespread bidirectional promoters are the major source of cryptic transcripts in yeast. Nature 457: 1038-1042.

Nicolas E, Yamada T, Cam HP, Fitzgerald PC, Kobayashi R, Grewal SI. 2007. Distinct roles of HDAC complexes in promoter silencing, antisense suppression and DNA damage protection. Nat Struct Mol Biol 14: 372-380.

Noma K, Allis CD, Grewal SI. 2001. Transitions in distinct histone $\mathrm{H} 3$ methylation patterns at the heterochromatin domain boundaries. Science 293: 1150-1155.

Pall GS, Codony-Servat C, Byrne J, Ritchie L, Hamilton A. 2007. Carbodiimide-mediated cross-linking of RNA to nylon membranes improves the detection of siRNA, miRNA and piRNA 
by northern blot. Nucleic Acids Res 35: e60. doi: 10.1093/ nar/gkm112.

Proudfoot N. 2004. New perspectives on connecting messenger RNA 3 ' end formation to transcription. Curr Opin Cell Biol 16: $272-278$.

Provost P, Silverstein RA, Dishart D, Walfridsson J, Djupedal I, Kniola B, Wright A, Samuelsson B, Radmark O, Ekwall K. 2002. Dicer is required for chromosome segregation and gene silencing in fission yeast cells. Proc Natl Acad Sci 99: 1664816653.

Rhind N, Russell P. 1998. Tyrosine phosphorylation of cdc2 is required for the replication checkpoint in Schizosaccharomyces pombe. Mol Cell Biol 18: 3782-3787.

Rhind N, Furnari B, Russell P. 1997. Cdc2 tyrosine phosphorylation is required for the DNA damage checkpoint in fission yeast. Genes Dev 11: 504-511.

Richard P, Manley JL. 2009. Transcription termination by nuclear RNA polymerases. Genes Dev 23: 1247-1269.

Russell P, Nurse P. 1986. cdc25+ functions as an inducer in the mitotic control of fission yeast. Cell 45: 145-153.

Sabatinos SA, Forsburg SL. 2010. Molecular genetics of Schizosaccharomyces pombe. Methods Enzymol 470: 759-795.

Sandberg R, Neilson JR, Sarma A, Sharp PA, Burge CB. 2008. Proliferating cells express mRNAs with shortened 3' untranslated regions and fewer microRNA target sites. Science 320: 1643-1647.

Shankaranarayana GD, Motamedi MR, Moazed D, Grewal SI. 2003. Sir2 regulates histone $\mathrm{H} 3$ lysine 9 methylation and heterochromatin assembly in fission yeast. Curr Biol 13: 1240-1246.

Singleton TL, Levin HL. 2002. A long terminal repeat retrotransposon of fission yeast has strong preferences for specific sites of insertion. Eukaryot Cell 1: 44-55.

Stoica C, Carmichael JB, Parker H, Pare J, Hobman TC. 2006. Interactions between the RNA interference effector protein Agol and 14-3-3 proteins: consequences for cell cycle progression. J Biol Chem 281: 37646-37651.

Sugiyama T, Cam H, Verdel A, Moazed D, Grewal SI. 2005. RNA-dependent RNA polymerase is an essential component of a self-enforcing loop coupling heterochromatin assembly to siRNA production. Proc Natl Acad Sci 102: 152-157.

Sun FL, Elgin SC. 1999. Putting boundaries on silence. Cell 99: $459-462$.

Tian B, Hu J, Zhang H, Lutz CS. 2005. A large-scale analysis of mRNA polyadenylation of human and mouse genes. Nucleic Acids Res 33: 201-212.

Verdel A, Jia S, Gerber S, Sugiyama T, Gygi S, Grewal SI, Moazed D. 2004. RNAi-mediated targeting of heterochromatin by the RITS complex. Science 303: 672-676.

Volpe TA, Kidner C, Hall IM, Teng G, Grewal SI, Martienssen RA. 2002. Regulation of heterochromatic silencing and histone $\mathrm{H} 3$ lysine-9 methylation by RNAi. Science 297: 1833-1837.

Volpe T, Schramke V, Hamilton GL, White SA, Teng G, Martienssen RA, Allshire RC. 2003. RNA interference is required for normal centromere function in fission yeast. Chromosome Res 11: 137-146.

West AG, Huang S, Gaszner M, Litt MD, Felsenfeld G. 2004. Recruitment of histone modifications by USF proteins at a vertebrate barrier element. Mol Cell 16: 453-463.

West S, Gromak N, Proudfoot NJ. 2004. Human 5' $\rightarrow$ 3' exonuclease Xrn2 promotes transcription termination at co-transcriptional cleavage sites. Nature 432: 522-525.

West S, Proudfoot NJ, Dye MJ. 2008. Molecular dissection of mammalian RNA polymerase II transcriptional termination. Mol Cell 29: 600-610.

Wilhelm BT, Marguerat S, Watt S, Schubert F, Wood V, Goodhead I, Penkett CJ, Rogers J, Bahler J. 2008. Dynamic repertoire of a eukaryotic transcriptome surveyed at single-nucleotide resolution. Nature 453: 1239-1243.

$\mathrm{Xu} \mathrm{Z,} \mathrm{Wei} \mathrm{W,} \mathrm{Gagneur} \mathrm{I,} \mathrm{Perocchi} \mathrm{F,} \mathrm{Clauder-Munster} \mathrm{S,}$ Camblong J, Guffanti E, Stutz F, Huber W, Steinmetz LM. 2009. Bidirectional promoters generate pervasive transcription in yeast. Nature 457: 1033-1037.

Zofall M, Grewal SI. 2006. Swi6/HP1 recruits a JmjC domain protein to facilitate transcription of heterochromatic repeats. Mol Cell 22: 681-692. 


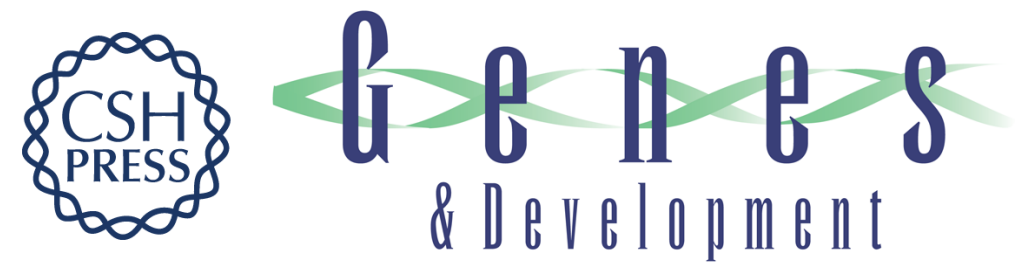

\section{Autoregulation of convergent RNAi genes in fission yeast}

Monika Gullerova, Danesh Moazed and Nick J. Proudfoot

Genes Dev. 2011, 25: originally published online February 28, 2011

Access the most recent version at doi:10.1101/gad.618611

\footnotetext{
Supplemental http://genesdev.cshlp.org/content/suppl/2011/02/18/gad.618611.DC1

Material

Related Content RNAi genes pave their own way

Leonie M. Kamminga and René F. Ketting

Genes Dev. March , 2011 25: 529-533

References This article cites 58 articles, 20 of which can be accessed free at: http://genesdev.cshlp.org/content/25/6/556.full.html\#ref-list-1

Articles cited in: http://genesdev.cshlp.org/content/25/6/556.full.html\#related-urls

License Freely available online through the Genes \& Development Open Access option.

Email Alerting Receive free email alerts when new articles cite this article - sign up in the box at the top Service right corner of the article or click here.
}

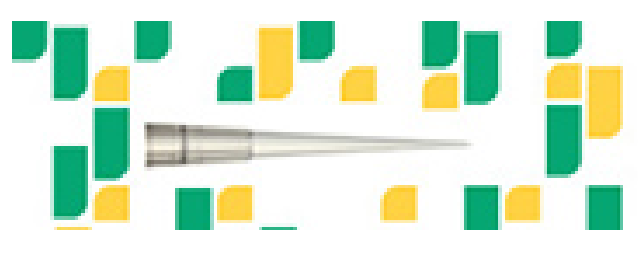

Focused on your science. 